\title{
Effects of a trail running competition on muscular performance and efficiency in well-trained young and master athletes
}

\author{
Christopher Schmidt Easthope ; Christophe Hausswirth ; Julien Louis ; Romuald Lepers ; Fabrice \\ Vercruyssen ; Jeanick Brisswalter \\ C. S. Easthope ; J. Louis ; J. Brisswalter : University of Nice Sophia Antipolis, Nice, France ; e-mail: brisswalter@unice.fr \\ C. Hausswirth : National Institute of Sports Expertise and Performance, Paris, France \\ R. Lepers : INSERM U887, Motricité Plasticité, Université de Bourgogne, Dijon, France \\ F. Vercruyssen : HandiBio, EA 4322, Université du sud Toulon-Var, Toulon, France
}

\begin{abstract}
To determine the acute effects of a trail running competition and the age-dependent differences between young and master athletes, 23 subjects [ 10 young ( $30.5 \pm 7$ years), 13 master (45.9 \pm 5.9 years)] participated in a 55-km trail running competition. The study was conceived as an intervention study compromising pre, post 1, 24, 48 and $72 \mathrm{~h}$ measurements. Measurements consisted of blood tests, ergometer cycling and maximal isometric voluntary contractions (MVC). Parameters monitored included MVC, twitch- and M-wave properties, EMG (RMS) of the vastus lateralis, two locomotion efficiency calculations and muscle damage markers in the blood (CK, LDH). Results indicate post-race increases in CK and $\mathrm{LDH}$, decreases in MVC values $(-32 \mathrm{vs}$. $-40 \%$ in young and master, $P<0.01)$, decreases in EMG, increases in contraction time and concomitant decreases in peak twitch values, and a decrease in locomotion efficiency ( -4.6 vs. $-6.3 \%$ in young and master, $P<0.05)$. Masters showed similar fatigue and muscle damage than young but recuperation was slowed in masters. This study shows that trail runs are detrimental to muscle function, and gives indication that training may not halt muscle deterioration through aging, but can help maintain performance level.
\end{abstract}

Keywords: Trail running / Ultra long distance /Master athlete / Eccentric contractions / Muscle damage / Efficiency

\section{Introduction}

While the popularity of running trail events has increased over the past 5 years (Hoffman and Wegelin 2009), limited information concerning the physiological responses of the tanner occurring during this type of contest is available. Trails can be defined as ultra-long-distance runs lasting over $5 \mathrm{~h}$ in duration which are performed in a mountain context, involving extensive vertical displacement (both uphill and downhill). One of the main performance determining components of trail runs is exercise duration. In general, ultra-endurance exercices such as marathon running, road cycling, or Ironman triathlons are well-known to impose a strenuous physical load on the organism, which leads to decreases in locomotion efficiency and concomitant substrate changes (Brisswalter et al. 2000; Fernström et al. 2007), thermal stress coupled with dehydration (Sharwood et al. 2004), oxidative stress (Nieman et al. 2004; Suzuki et al. 2006), and specifically in running events, structural muscle damage (Overgaard et al. 2004; Suzuki et al. 2006). The second major characteristic of trail running events is the large proportion of eccentric work performed during the downhill segments of the race. Eccentric contractions involve force generation in a lengthening muscle, and are known to procure severe structural damage in muscles, affecting their contractile and recuperative properties (Nicol et al. 2006). Several studies in the last decade have investigated the effects of long-distance runs performed on level courses. Collective results show an increased release of muscular enzymes into the plasma, a structural disruption of the sarcomere, a substantial impairment in maximal force generation capacity (Lepers et al. 2000a; Millet et al. 2002, 2003; Overgaard et al. 2004; Place et al. 2004), and a decrease in post-race locomotion efficiency (Millet et al. 2000, 2009), indicating that muscles are progressively damaged during the exercise. Specifically, maximal isometric knee extension force has been reported to decrease by $24 \%$ after a $30-\mathrm{km}$ running race (Millet et al. 2003), by $28 \%$ after $5 \mathrm{~h}$ of treadmill running (Place et al. 2004) and by $30 \%$ after a $65-\mathrm{km}$ ultra-marathon (Millet et al. 2002). Recently, 
Millet et al. (2009) reported a 6.2\% decrease in running efficiency 3 weeks after a 8,500-km run between Paris and Beijing performed in 161 days. Gauché et al. (2006) have reported that maximal voluntary force decreased by $37 \%$ at the end of a prolonged trail run. Repeated eccentric contractions may also affect locomotion efficiency, as demonstrated by Braun and Dutto (2003), who observed a decrease of $3.2 \%$ in running efficiency $48 \mathrm{~h}$ after a 30 -min downhill run. In a similar vein, Moysi et al. (2005) found a $6 \%$ decrease in cycling efficiency after 10 series of 25 repetitions of squats, an eccentric exercise. Repeated eccentric contractions, independent of their context, seem to induce a decrease in locomotion efficiency, even if efficiency is evaluated in concentrically dominated cycling (Moysi et al. 2005). Based upon the reviewed literature, it was assumed that trail running races would accentuate muscle damage when compared to level running, due to the large proportion of eccentric contractions occurring in the successive downhill segments of courses and therefore lead to both a decrease in muscular performance and locomotion efficiency. Few studies so far have analyzed the physiological aspects of trail running. The existing studies mainly focused on the origin of the decline in contraction capacity (e.g. Miles et al. 2006; Gauché et al. 2006) or on pacing strategies during the race (Stearns et al. 2009). To our knowledge, only limited data is available on the impact of this type of events on locomotion efficiency (Millet et al. 2000).

A further characteristic of trail running competitions is the increasing participation of master athletes (Hoffman and Wegelin 2009). Tanaka and Seals defined master athletes in their 2008 article as individuals who regularly participate in endurance training and who try to maintain their physical performance level despite the aging process. In a competition context, competitors are traditionally classified as master athletes when over 40 years of age, the age at which a first decline in endurance peak performance is observed (Lepers et al. 2010; Sultana et al. 2008; Tanaka and Seals 2008). The aging process induces a great number of structural and functional transformations, which lead to an overall decline in physical capacity (Thompson 2009). The current rising of the average age in western countries has procured the need to design strategies which increase functional capacity in older people and so forth ameliorate the standard of living (e.g. Henwood and Taaffe 2006). Observing master athletes can give insight into age-induced changes in physiology and adaptability, thus enabling scientists to develop more concise and effective recuperation and mobilization programs. Recent studies have shown that master endurance athletes are able to maintain their performance despite exhibiting the structural changes in muscle performance and in maximal aerobic power which are classically associated with aging (Lepers et al. 2010; Tanaka and Seals 2008; Bieuzen et al. 2010; Louis et al. 2009).

In this context, the first purpose of our study was to evaluate muscle performance and efficiency of runners participating in a long-distance trail competition. Trail running was expected to procure important muscle damage and decrease in locomotion efficiency. The second purpose was to compare changes in these parameters between young and master participants. We hypothesized that neuromuscular alterations following the competition would be greater in master athletes compared to young athletes.

\section{Materials and methods}

\section{Subjects}

Eleven young and fifteen master athletes, all well-motivated, volunteered to participate in this study. The characteristics of the subjects are shown in Table 1. All subjects had to be free from present or past neuromuscular and metabolic conditions that could have affected the recorded parameters. The subjects had regular training experience in long-distance running prior to the study $(8.4 \pm 6.0$ years for the young vs. $13.3 \pm 7.8$ years for the master runners), and had performed a training program of $72.1 \pm$ 25.1 and $74.1 \pm 23.6 \mathrm{~km} /$ week, respectively, for young and masters during the 3 months preceding the experiment. The local ethics committee (St Germain en Laye, France) reviewed and approved the study before its initiation and all subjects gave their informed written consent before participation. 
The study was divided into four phases; preliminary testing and familiarization, pre-testing, trail race intervention and post-testing (see Fig. 1). During the first phase, subjects were familiarized with the test scheme and location, and preliminary tests were performed. During the third phase, subjects performed a $55-\mathrm{km}$ trail running race in a medium altitude mountain context. During the second and the fourth phases, muscle performance and efficiency were analyzed and blond samples were collected. All physiological parameters were recorded 1 day before (pre) and 3 days after the trail running race (post 1, 24, 48, and $72 \mathrm{~h}$ ).

\section{Preliminary session}

During a preliminary session that took place 1 month before the experiment, 26 subjects (11 young and 15 masters) underwent an incremental cycling test at a self-selected cadence on an electromagnetically braked ergocycle (SRM, Schoberer Rad Messtechnik, Jülich, Welldorf, Germany). In accordance with the recommendations of the ethic committee and the French Medical Society, a cycle ergometer protocol was chosen to evaluate ventilatory parameters and efficiency, even though a running protocol would have been preferred. Considerations were based on the assumption that extremely fatigued subjects would have difficulties running on a treadmill and that this might lead to injuries. Moysi et al. (2005) have shown that eccentric muscle damage affects locomotion efficiency and ventilatory parameters in a cycling protocol in a similar way to a running protocol. The ergocycle allows subjects to maintain a constant power output which is independent of the selected cadence, by automatically adjusting torque to angular velocity. The test consisted of a warm-up lasting $6 \mathrm{~min}$ at $100 \mathrm{~W}$, and an incremental period in which the power output was increased by $30 \mathrm{~W}$ each minute until volitional exhaustion. During this incremental cycling exercise, oxygen uptake $\left(\mathrm{VO}_{2}\right)$, minute ventilation (VE), and respiratory exchange ratio (RER) were continuously measured every $15 \mathrm{~s}$ using a telemetric system (Cosmed K4b2, Rome, Italy). The criteria used for the determination of VO2max were a plateau in $\mathrm{VO}_{2}$ despite an increase in power output, a RER above 1.1, and a heart rate (HR) above $90 \%$ of the predicted maximal HR (Howley et al. 1995). Maximal oxygen uptake $\left(\mathrm{VO}_{2 \max }\right)$ was determined as the average of the last three highest $\mathrm{VO}_{2}$ values recorded $(58.8 \pm 6.5 \mathrm{ml} / \mathrm{min} / \mathrm{kg}$ for the young vs. $55.0 \pm$ $5.8 \mathrm{ml} / \mathrm{min} / \mathrm{kg}$ for the master athletes). The ventilatory threshold (VT) was determined according to the method described by Wasserman et al. (1973). The maximal aerobic power output (MAP) was the highest power output completed in $1 \mathrm{~min}(352.5 \pm 41.1 \mathrm{~W}$ for the young vs. $347.6 \pm 62.9 \mathrm{~W}$ for the master athletes).

\section{Race conditions}

The running event was a 55-km trail race involving a 6,000 $\mathrm{m}$ vertical displacement $(3,000-\mathrm{m}$ up and 3,000-m down). The starting point and finishing line were at 694-m altitude, and the highest point of the race was at $3,050 \mathrm{~m}$. Due to the competitive nature of the intervention, each subject was well motivated to perform maximally over the distance. From the initial group (11 young and 15 masters), only three subjects (one young and two master athletes) did not finish the course. Therefore, all data presented corresponds to the finalist group (10 young and 13 master athletes). Physical activity after the race was controlled (walking activities were limited and massages were prohibited). Mean race times performed by subjects are shown in Table 1 .

\section{Maximal isometric force and muscle properties}

Ten minutes after the sub-maximal cycling exercise, the maximal voluntary isometric force of the right knee extensor (KE) muscles was determined using an isometric ergometer chair (type: J. Sctnell, Selephon, Germany) connected to a strain gauge (Type: Enertec, schlumberger, Villacoublay, France). Subjects were comfortably seated and the strain gauge was securely connected to the right ankle. The angle of the right knee was fixed at $100^{\circ}\left(0^{\circ}=\right.$ knee fully extended $)$. Extraneous movement of the upper body was limited by two harnesses enveloping the chest and the abdomen. For each testing session, the subjects were asked to perform three $2-3 \mathrm{~s}$ maximal isometric contractions $(0 \mathrm{rad} / \mathrm{s})$ of the KE muscles. The subjects were verbally encouraged and the three trials were executed with a 1-min rest period. The trial with the highest force value was selected as the maximal isometric voluntary contraction (MVC, in Newton). In addition to MVC, the M-wave of the vastus lateralis was recorded 
from a twitch evoked by an electrical stimulation. Changes in neuromuscular properties were evaluated throughout all the testing sessions (Lepers et al. 2000b; Place et al. 2004). Electrical stimulation was applied to the femoral nerve of the dominant leg according to the methodology previously described by Place et al. (2004). The following parameters of the muscular twitch were obtained: (a) peak twitch $(\mathrm{Pt})$, i.e. the highest value of twitch tension production and $(\mathrm{b})$ contraction time $(\mathrm{Ct})$, i.e. the time from the origin of the mechanical response to Pt.

\section{EMG recordings}

During the MVC, electrical activity of the vastus lateralis (VL) muscle was monitored using bipolar surface electrodes (Blue sensor Q-00-S, Medicotest SARL, France). The pairs of pregelled Ag/AgC1 electrodes (interelectrode distance $=20 \mathrm{~mm}$; area of electrode $=50 \mathrm{~mm}^{2}$ ) were applied along the fibers at the height of the muscle belly, as recommended by the SENIAM. A low skin impedance $(<5 \mathrm{k} \Omega)$ was obtained by abrading and cleaning the area with an alcohol wipe. The impedance was subsequently measured with a multimeter (Isotech, IDM 93N). To minimize movement artifacts, the electrodes were secured with surgical tape and cloth wrap. A ground electrode was placed on a bony site over the right anterior superior spine of the iliac crest. To ensure that the electrodes were precisely at the same place for each testing session, the electrode location was marked on the skin with an indelible marker. EMG signals were pre-amplified (Mazet Electronique Model, Electronique du Mazet, Mazet Saint-Voy, France) close to the detection site (common-mode rejection ratio $=100 \mathrm{~dB} ; Z$ input $=$ $10 \mathrm{G} \Omega$; gain $=600$; bandwidth frequency $=6-1,600 \mathrm{~Hz}$ ). EMG data were sampled at 1,000 $\mathrm{Hz}$ and quantified by using the root mean square (RMS). Maximal RMS EMG of VL muscle was set as the maximal 500-ms RMS value found over the 3-s MVC (i.e.,500 ms window width, 1-ms overlap) using the propriety software Origin 6.1. During evoked stimulation performed before the MVC, peak-to-peak amplitude (PPA) and peak-to-peak duration (PPD) of the M-wave were determined for the VL muscle. Amplitude was defined as the sum of absolute values for maximum and minimum points of the biphasic (one positive and one negative deflection) M-wave. Duration was defined as the time from maximum to minimum points of the biphasic M-wave.

Blood markers of muscle damages

For each evaluations series, $15 \mathrm{ml}$ of blood was collected into vacutainer tubes via antecubetal venipuncture. The pre-exercise sample was preceded by a 10-min rest period. Once the blood sample was taken, tubes were shuffled by turning and placed on ice for $30 \mathrm{~s}$ before centrifugation $(10 \mathrm{~min}$, $3,000 \mathrm{~T} / \mathrm{min}, 4^{\circ} \mathrm{C}$ ). The obtained plasma sample was then stored in multiple aliquots (Ependorf type, $500 \mu \mathrm{l}$ per samples) at $-80^{\circ} \mathrm{C}$ until analyzed for the markers described below. All assays were performed in duplicate on first thaw. As a marker of sarcolemma disruption, the activity of the muscle enzymes creatine kinase (CK) and lactodeshydrogenase (LDH) were measured spectrophotometrically in the blood plasma using commercially available reagents (Roche/Hitachi, Meylan, France).

\section{Locomotion efficiency}

Subjects were asked to perform a cycling control exercise (CTRL) at a self-selected cadence on the same ergocycle as used in the preliminary session. This cycling exercise involved 6 min at $100 \mathrm{~W}$ followed by $10 \mathrm{~min}$ at a relative power output corresponding to the ventilatory threshold. For each subject and each cycling session, metabolic data was continuously recorded to assess the efficiency in cycling.

Efficiency can be expressed as a ratio between (external) power output and the ensuing energy expenditure (EE). Efficiency may, however, be calculated in a variety of ways (Martin et al. 2005). In this study, two types of efficiency calculation were employed, gross efficiency (GE), and delta efficiency (DE). GE is defined as work rate divided by energy expenditure and calculated using the following equation (Gaesser and Brooks 1975):

Gross efficiency $(\%)$ 


$$
=\frac{\text { Work rate }(\mathrm{WR}, \text { in joules })}{\text { Energy expenditure (EE, in joules) }} \times 100
$$

DE is considered by many to be the most valid estimate of muscular efficiency (Gaesser and Brooks 1975; Coyle et al. 1992). DE calculations are based upon a series of work rates which are then subjected to linear regression analysis. In this study, work rates were calculated from the two intensity tiers completed in the test described at the beginning of this section.

Delta efficiency (\%)

$$
=\frac{\Delta \text { Work rate }(\mathrm{WR}, \text { in joules })}{\Delta \text { Energy expenditure (EE, in joules) }} \times 100
$$

In order to obtain precise values for work rate utilized in the efficiency calculations, power output was assessed from the set work rate and the true cadence as monitored by the SRM crank system. EE was obtained from the rate of oxygen uptake, using the equations developed by Brouwer (1957). These equations take the substrate utilization into account, by calculating the energetic value of oxygen based on the RER value. To minimize a potential influence of the $\mathrm{VO}_{2}$ slow component, which might vary between subject groups, the mean EE during the 3rd to 6th minute was used in the calculations of GE and DE.

\section{Statistical analysis}

All data presented are mean \pm SD (tables and figures). Each dependent variable was then compared between the different testing conditions using a two-way ANOVA with repeated measures (period vs. group). Newman-Keuls post-hoc tests were applied to determine the between-mean differences, if the analysis of variance revealed a significant main effect for period or interaction of group $\mathrm{x}$ period. For all statistical analysis, a $P<0.05$ value was accepted as the level of significance.

\section{Results}

Muscular performance

In all evaluations, MVC values of master athletes were significantly lower than the young group' s values $(-21.8 \pm 4.6 \%, P<0.01)$. One hour after the intervention (post), maximal isometric strength values of knee extensors decreased significantly when compared with pre-race values, in nonsignificant different proportions for young $(-32 \%, P<0.01)$ and master athletes $(-40 \%, P<0.01)$. MVC values for young subjects returned to baseline at post $24 \mathrm{~h}$, at which time the MVC reduction in masters remained significant $(-13.6 \%, P=0.04)$. A significant decrease in EMG activity (RMS) during MVC of the vastus lateralis (VL) muscle was observed at 1 and $24 \mathrm{~h}$ post-exercise without any differences between groups or periods. Compared with pre-race values, post-exercise MVC RMS values decreased in young by $-40.2 \pm 19 \%$ and in masters by $-42 \pm 19.2 \%(P<0.01)$ (Fig. 2$)$.

Muscular twitch and M-wave properties

Before the race, no significant effect of age was observed on peak twitch torque (Pt) or contraction time $(\mathrm{Ct})$. One hour after the race, no effect was recorded on $\mathrm{Ct}$ or Pt independent of the group. Post 24 $\mathrm{h}$, a slower contraction time $(\mathrm{Ct})$ and a lower peak twitch torque $(\mathrm{Pt})$ were recorded in both groups. Compared to pre-race values, Pt decreased by $18.2 \%(P=0.04)$ in young and by $23.5 \%(P=0.02)$ in master runners at post $24 \mathrm{~h}$. These alterations in twitch properties returned close to pre-test values in young subjects, but remained significant for $48 \mathrm{~h}(P=0.03)$ and $72 \mathrm{~h}(P=0.04)$ in masters subjects (Table 2).

Before the race, no significant effect of age was observed on PPA or PPD values of the M-wave for the VL muscle (Table 2). One hour after the race, a significant increase in PPD was observed in both 
groups. This increase remained significant in the master athlete group at post $24 \mathrm{~h}(P=0.02)$, while the young group returned close to baseline values. Furthermore, in masters, PPA values decreased below pre-race values $48 \mathrm{~h}(P=0.04)$ and $72 \mathrm{~h}(P=0.02)$ after the race, while no effects were observed in young subjects.

Blood markers of muscle damages

Twenty-four hours (Post $24 \mathrm{~h}$ ) after the race, the plasma activities of CK and LDH increased significantly in comparison to pre-race values, with a greater increase for master subjects $(P=0.04)$. CK and LDH values remained significantly elevated at post $48 \mathrm{~h}$ and post $72 \mathrm{~h}$, without any difference between groups (Table 3).

Locomotion efficiency and cycling cadence

Gross efficiency (GE), delta efficiency (DE) and cadence values are presented in Table 4. No significant difference in GE, DE or cadence was observed between groups before the race. After the race, results indicated a non-group specific, significant decline in GE from post 24 to $72 \mathrm{~h}$ (GE mean decrease in young vs. masters in $\%$ of pre-race values: -4.7 vs. $-6.3 \%$, respectively). In both groups, VE increased post $24,48,72 \mathrm{~h}$ in comparison to pre-test values (VE mean increase in young vs. masters in $\%$ of pre-race values: +11.7 vs. $+10.1 \%$, respectively). No significant change in DE was observed in young subjects alter the race. On contrary, a significant decrease in DE was recorded in master subjects (DE decrease in master athletes of post $24 \mathrm{~h}(P=0.02)$, post $48 \mathrm{~h}(P=0.01)$ and post $78 \mathrm{~h}(P=0.03)$ in $\%$ of pre-race values: $-10.6,-10.4,-11.5 \%$, respectively).

Post-race cadence was significantly higher in all post-race evaluations for young subjects when compared with masters. Results indicate a significant increase in cycling cadence post $24 \mathrm{~h}(+4.4 \%, P$ $=0.04)$, post $48 \mathrm{~h}(+10.6 \%, P=0.03)$, and post $72 \mathrm{~h}(+17 \%, P<0.01)$ for young, and only in post 48 $\mathrm{h}(+3.9 \%, P=0.04)$ and post $72 \mathrm{~h}(+10.8 \%, P=0.03)$ for master athletes.

\section{Discussion}

The objective of the present study was to investigate changes in muscular performance and locomotion efficiency in well-trained endurance runners procured through a trail running competition and to compare these to literature data taken from level courses. The participation of two different age groups of runners (young vs. masters) allowed additional study of the effect of age on fatigue generation and recuperation. The main results of our study indicate that: (1) post-run muscular performance and locomotion efficiency decline, while the associated concentrations of muscle damage indicating blood markers rise, regardless of age, and (2) changes of proportion of the differences between groups are only visible in the recuperation phase (post 24-72 h).

The running event analyzed in this study was a $55-\mathrm{km}$ trail race featuring a $6,000-\mathrm{m}$ vertical displacement (3,000-m up and 3,000-m down). The average race time was 06:45 + 00:45. As stated, the main performance components of trail running are exercise duration and vertical displacement (uphill and downhill). From this perspective, trail running competitions induce an intensive physical work load on the organism. Considering the popularity of trail running and the abundance of competitions over the world, it appears important to precisely characterize the acute physiological reactions consecutive to such events.

One of the most significant consequences of the race was a reduction in muscular performance. The recorded data manifests a significant decline in maximal force-generating capacities in young $(-32 \%)$ and master athletes $(-40 \%) 1 \mathrm{~h}$ post-race. The intervention seems to have decreased MVC in a slightly greater magnitude compared to: (i) prolonged run on level such as 5 -h treadmill running (-28\%, Place et al. 2004) or shorter $30-\mathrm{km}$ trait race $(-24 \%$, Millet et al. 2003) or race of longer duration but with lower altitude change (-30\%, Millet et al. 2002). An adaption for workload distribution, age, training status and a methodological standardization would have to be conducted before a precise comparison between races is possible. It is generally accepted though, that the structural muscle damage leading to 
MVC loss is generated by the eccentric muscle contractions occurring in running (Millet et al. 2002, 2003; Overgaard et al. 2004; Place et al. 2004). As a logical consequence, interventions with a greater overall percentage of eccentric force production should induce a more pronounced MVC decline in comparison to level courses of same duration, in which the eccentric component is not as pronounced: the recorded MVC data of this study thus conforms to this idea. Additional studies will need to clarify the relative contribution of duration versus altitude changes to the decrease of muscular capacities following prolonged running exercices.

After the race (post 24-72 h), MVC values progressively returned to their pre-race level. In addition, results indicate a significant decrease in VL muscle activity (i.e. RMS values) recorded during MVC performed $1 \mathrm{~h}$ after the race which persisted until $72 \mathrm{~h}$ after the race. Further parameters used to characterize muscular fatigue included muscular twitch and $\mathrm{M}$-wave properties. $\mathrm{Pt}$ decreased significantly $24 \mathrm{~h}$ after the race, accompanied by a concomitant increase in $\mathrm{Ct}$ from 24 to $72 \mathrm{~h}$ after the race, albeit only in masters. The main explanation for these perturbations of contractile parameters could be an alteration of the excitation-contraction coupling process that can be attributed to several mechanisms including, but not limited to, reduced $\mathrm{Ca}^{2+}$ release from the sarcoplasmic reticulum (Westerblad et al. 1991), a decrease in blood $\mathrm{pH}$ and a reduced rate or force of crossbridge latching (Metzger and Moss 1990). An increase in Ct after the race could also indicate an impairment in type II muscles fibers (i.e. fast contraction fibers) which may be compensated for by the more fatigue resistant type I muscle fibers (i.e. slow contraction fibers). Twitch muscle properties were unchanged at 1-h post-race, alterations appearing only $24 \mathrm{~h}$ after the race and later. This phenomenon might suggest that muscle fatigue was counterbalanced by potentiation mechanisms occurring immediately after the race (Baudry et al. 2005; Shima et al. 2007; Bieuzen et al. 2009). By contrary, M-wave PPD was significantly reduced immediately after the race (post) and tended to return to basal values 24-72 $\mathrm{h}$ after the race. The master group exhibited increased M-wave PPA at 24-h post-race. As previously described in the literature, these increases in M-wave parameters suggest an alteration in muscle excitability; probably generated by impairments in neuromuscular propagation due to an increase in sarcolemma permeability to sodium, potassium and chloride (Lepers 2009). These results support the assumption of muscle damage development through trail running. The data recorded for muscle damage indicating blood markers underscores this observation. A post-race increase in the plasma activity of muscle enzymes (CK and LDH), which persisted for several days after the race (Table 3), was recorded. Similarly, Suzuki et al. (2006) reported a significant increase in CK and LDH activities in the plasma soon after an Ironman triathlon, which remained elevated until 1 day after the race. Intracellular enzymes such $\mathrm{CK}$ and $\mathrm{LDH}$ indicate that the muscle injury arises from myofibrillar disruption (Clarkson et al. 1992; Noakes 1987), and are classically used to assess the loss of sarcolemmal integrity after strenuous exercices (Overgaard et al. 2004). As neither CK nor LDH is considered redundant indicators (Warren et al. 1999), the analysis was augmented by the acquisition of further physiological variables.

As an important determinant of performance in endurance events, locomotion efficiency is classically surveyed in athletes in order to evaluate the effects of particular training periods (Santalla et al. 2009). It has been reported that even small increments in cycling efficiency may lead to major improvements in endurance performance (Moseley and Jeukendrup 2001). The efficiency of physical work is a measure of the body' s effectiveness in converting chemical energy into mechanical energy. Efficiency was here calculated as described in the methods section; the quotient of work rate and energy expenditure (Gaesser and Brooks 1975). A decrease in locomotion efficiency can therefore be interpreted as either a relative increase in energy expenditure, or a relative decrease in work rate. Considering that work rate was fixed for all the tests, increased energy expenditure remains the only viable option. Recorded values show a decline in GE in both groups of athletes after the race, which persisted until $72 \mathrm{~h}$ post-race. Although commonly employed, GE has been criticized for its inclusion of energy-delivery processes that do not contribute to production of mechanical work in the denominator. Therefore, locomotion efficiency was also evaluated through DE calculation, which is by many considered to be the most valid estimate of muscular efficiency (Gaesser and Brooks 1975; Coyle et al. 1992; Mogensen et al. 2006). While GE values declined for both groups, DE values only declined in the masters group (P24, P48, and P72), thus confirming the increase in energy expenditure to ensure a continuous power output. This phenomenon is largely related to a decline in muscular performance. In order to produce the same locomotive work as in the pre-race condition, strategies 
such as an increase in spatio-temporal recruitment of muscle fibers or an increase in cycling cadence, involving a concomitant increase in VE (Table 4) could be engaged. The attained results provide evidence of an alteration of cycling efficiency in both groups tested.

The second aim of this study was to analyze age-related effects on muscular performance and cycling efficiency after the trail race by comparing physiological variables recorded in young and master athletes. Race completion time did not significantly differ between groups (06:42 + 00:51 vs. 06:51 + 00:47, for young vs. masters, respectively). Despite the structural and functional alterations typically observed during the aging process, master athletes were able to produce the same level of performance as the young group. This observation confirms the realistic possibility of preventing the age-related decline of physical performance through physical activity.

The analysis of muscular performance in the two groups of athletes shows a classical decline in maximal force-generating capacity in masters $(-21.8 \pm 4.6 \%)$, when compared with young for all testing sessions performed before and after the race (Louis et al. 2009; Bieuzen et al. 2009). Results additionally indicate a similar decrease in MVC values at 1-h post-race in both age groups which, in the master subjects only, persisted until $24 \mathrm{~h}$ after the race, suggesting a slower recovery. Based on the results of Coggan et al. (1990), which were confirmed by Tarpenning et al. (2004), the age-induced decrease of MVC values in master athletes similar to our experimental population can be mainly explained by neural factors such as muscle recruitment and/or specific tension. The twitch analysis based assessment of muscular function seems to confirm this hypothesis. This study is to our knowledge the first to present twitch and $\mathrm{M}$-wave data for master athletes after a trail running competition. As previously described in studies on long-distance exercise induced fatigue in young subjects (e.g. Millet et al. 2002), Pt and Ct parameters increased $24 \mathrm{~h}$ after the race. The proportions were similar in both groups tested. The alterations in muscular properties persisted several days after the race in masters only, further supporting the idea of a slower muscle recovery in this group. Master M-wave PPD values increased proportionally to the aforementioned development in the young group at 1-h post-race, and returned to pre-race values in all the following testing conditions. By contrary, master M-wave PPA values decreased significantly from 48 to $72 \mathrm{~h}$ after the race, while this decline was marginal in young athletes. Despite a similar training status in young and master athletes, the values of these parameters show a greater alteration in masters' muscular function (i.e. contractility and excitability) after the race, indicating a slower recovery of muscle strength. An assessment of VL muscle activity shows that this effect is not brought by an age-induced muscle activation impairment, as MVC RMS values declined in similar proportions between groups after the race.

As depicted in Table 3, CK and LDH activity in plasma increased in similar proportions after the race for both groups indicating a similar level of muscular deterioration between groups following the trail competition. This is in line with the above mentioned results, as the proportion of the competitioninduced reduction of MVC was similar between groups.

This might support the idea that regular endurance training reinforces active muscles, and therefore limits the structural and functional changes classically associated with aging (Lexell 1995).

Results of this study show an effect of aging on cycling efficiency before and after the running race. While GE declined in similar proportions in both groups after the race, DE declined only in masters 24, 48 and $72 \mathrm{~h}$ after the race (Table 4). The GE decline in both groups could be mainly related to increases in energy-delivery processes that do not contribute to mechanical work. Variations in these processes originate through modifications in cycling kinematics (e.g. cycling cadence) or muscular contraction patterns (e.g. recruitment of subsidiary muscles, increase in antagonistic co-activation) in fatigued muscles and must be considered when regarding the GE (Braun and Dutto 2003). The decline of $\mathrm{DE}$ in masters could be strongly related to alterations in muscular performance, provoking an increase in muscle activity in cycling to produce the same external work. Gleeson et al. (1998) suggested that an increase in type II fiber recruitment may occur when exercise is performed in a fatigued state. In addition, if force-generating capacity was compromised, more motor units would have to be activated to achieve the same submaximal force output, resulting in a concomitant increase in metabolic cost (Braun and Dutto 2003). Such an effect could contribute to the significantly higher 
VE shown in the present study. The results demonstrate that master athletes reached a similar level of fatigue through the race, when compared to young athletes, but recovered significantly slower. The hypothesis that master athletes achieve a higher level of fatigue through similar exertion can therefore be no longer supported. This was surprising, as the input parameters of master athletes were considerably lower and therefore either a lower performance or a greater fatigue would be expected. Thus, it must be surmised that masters must in some form economize energy expenditure over the length of the course, for example through adaptations in strategy or locomotor patterns.

\section{Conclusion}

The aim of this study was to assess physiological responses to an exhaustive trail running competition and to analyze possible differences between young and master athletes.

A 55-km ultra-endurance event was used as a fatigue generating intervention. An especially large amount of muscular fatigue was generated through the large proportion of eccentric contractions occurring during the downhill sections of the race. Results indicate an acute fatigue in all subjects (young and masters), which is mainly represented by decreases in muscle performance. Despite similar race performances between groups, the generated fatigue was similar between groups. Post-race development of CK, and neuromuscular properties suggests a decrease in the recuperation kinetics of the master subjects. The results attained in this study give indication that regular endurance training cannot halt the age-related decline in muscle performance, but that performance level can non-the-less be maintained by global or local strategy adaptations or to-date unknown adaptations on a physiological level.

\section{References}

Baudry S, Klass M, Duchateau J (2005) Postactivation potentiation influences differently the nonlinear summation of contractions in young and elderly adults. J Appl Physiol 98:1243-1250

Bieuzen F, Hausswirth C, Louis J, Brisswalter J (2010) Age-related changes in neuromuscular function and performance following a high-intensity intermittent task in endurance-trained men. Gerontology $56: 66-72$

Braun WA, Dutto DJ (2003) The effects of a single bout of downhill running and ensuing delayed onset of muscle soreness on running economy performed 48 h later. Eur J Appl Physiol 90:29-34

Brisswalter J, Hausswirth C, Vercruyssen F, Collardeau M, Vallier JM, Lepers R, Goubault C (2000) Carbohydrate ingestion does not influence the charge in energy cost during a 2-h run in welltrained triathletes. Eur J Appl Physiol 81(1-2):108-113

Brouwer E (1957) One simple formulate for calculating the heat expenditure and the quantities of carbohydrate and fat oxidized in metabolism of men and animals from gaseous exchange (oxygen intake and calorie acid output) and urine-N. Acta Physiol Phannacol Neerl 6:795-802

Clarkson PM, Nosaka K, Braun B (1992) Muscle function after exercise-induced muscle damage and rapid adaptation. Med Sci Sports Exerc 24:512-520

Coggan AR, Spina RJ, Rogers MA, King DS, Brown M, Nemeth PM, Holloszy JO (1990) Histochemical and enzymatic characteristics of skeletal muscle in master athletes. J Appl Physiol 68:1896-1901

Coyle EF, Sidossis LS, Horowitz JF, Beltz JD (1992) Cycling efficiency is related to the percentage of type I muscle fibers. Med Sci Sports Exerc 24(7):782-788

Fernström N, Bakkman L, Tonkonogi M, Shabalina IG, Rozhdestvenskaya Z, Mattsson CM, Enqvist JK, Ekblom B, Sahlin K (2007) Reduced efficiency, but increased fat oxidation, in mitochondria from 
human skeletal muscle after 24-h ultraendurance exercise. J Appl Physiol 102(5):1844-1849

Gaesser GA, Brooks GA (1975) Muscular efficiency during steady-rate exercise: effects of speed and work rate. J Appl Physiol 38(6):1137-1139

Gauché E, Lepers R, Rabita G, Leveque J-M, Bishop D, Brisswalter J, Hausswirth C (2006) Vitamin and mineral supplementation and neuromuscular recovery after a running race. Med Sci Sports Exerc 38(12):2110-2117

Gleeson M, Blannin AK, Walsh NP, Field CN, Pritchard JC (1998) Effect of exercise-induced muscle damage on the blood lactate response to incremental exercise in humans. Eur J Appl Physiol Occup Physiol 77:292-295

Henwood TR, Taaffe DR (2006) Short-term resistance training and the older adult: the effect of varied programmes for the enhancement of muscle strength and functional performance. Clin Physiol Funct Imaging 26:305-313

Hoffman MD, Wegelin JA (2009) The Western States 100-Mile Endurance Run: participation and performance trends. Med Sci Sports Exerc 41:2191-2198

Howley ET, Bassett DR, Welch HG (1995) Criteria for maximal oxygen uptake: review and commentary. Med Sci Sports Exerc 27:1292-1301

Lepers R (2009) Muscle fatigue following prolonged dynamic exercise. In: Shinohara M (ed) Advances in neuromuscular physiology of motor skills and muscle fatigue. Research Signpost, Trivandrum, pp 369390

Lepers R, Pousson M, Maffiuletti N, Martin A, Van Hoecke J (2000a) The effects of a prolonged running exercise upon strength characteristics. Int J Sports Med 21:275-280

Lepers R, Hausswirth C, Maffiuletti N, Brisswalter J, Van Hoecke J (2000b) Evidence of neuromuscular fatigue after prolonged cycling exercise. Med Sci Sports Exerc 32(11):1880-1886

Lepers R, Sultana F, Bernard T, Hausswirth C, Brisswalter J (2010) Age related changes in triathlon performances. Int J Sport Med 31:251-256

Lexell J (1995) Human aging, muscle mass, and fiber type composition. J Gerontol A Biol Sci Med Sci 50:11-16

Louis J, Hausswirth C, Bieuzen F, Brisswalter J (2009) Muscle strength and metabolism in master athletes. Int J Sports Med 30(10):754-759

Martin DT, Quod MJ, Gore Ci, Coyle EF (2005) Has Armstrong's cycle efficiency improved? J Appl Physiol 99:1628-1629

Metzger JM, Moss RL (1990) Effects of tension and stiffness due to reduced $\mathrm{pH}$ in mammalian fast- and slow-twitch skinned skeletal muscle fibres. J Physiol 428:737-750

Miles MP, Walker EE, Conant SB, Hogan SP, Kidd JR (2006) Carbohydrate influences plasma interleukin6 but not C-reactive protein or creatine kinase following a 32-km mountain trail race. Int J Sport Nutr Exerc Metab 16:36-46

Millet G, Lepers R, Lattier G, Martin V, Babault N, Maffiuletti N (2000) Influence of ultra-long term fatigue on the oxygen cost of two types of locomotion. Eur J Appl Physiol 83:376-380 
Millet GY, Lepers R, Maffiuletti NA, Babault N, Martin V, Lattier G (2002) Alterations of neuromuscular function after an ultramarathon. J Appl Physiol 92:486-492

Millet GY, Martin V, Lattier G, Ballay Y (2003) Mechanisms contributing to knee extensor strength loss after prolonged running exercise. J Appl Physiol 94:193-198

Millet GY, Morin JB, Degache F, Edouard P, Feasson L, Verney J, Oullion R (2009) Running from Paris to Beijing: biomechanical and physiological consequences. Eur J Appl Physiol 107: 731-738

Mogensen M, Bagger M, Pedersen PK, Fernstrom M, Sahlin K (2006) Cycling efficiency in humans is related to low UCP3 content and to type I fibres but not to mitochondrial efficiency. J Physiol 571:669681

Moseley L, Jeukendrup AE (2001) The reliability of cycling efficiency. Med Sci Sports Exerc 33:621-627

Moysi JS, Garcia-Romero JC, Alvero-Cruz JR, Vicente-Rodriguez G, Ara I, Dorado C, Calbet JAL (2005) Effects of eccentric exercise on cycling efficiency. Can J Appl Physiol 30(3):259-275

Nicol C, Avela J, Komi PV (2006) The stretch-shortening cycle: a model to study naturally occurring neuromuscular fatigue. Sports Med 36:977-999

Nieman DC, Henson DA, McAnulty SR, McAnulty L, JD M, Ahmed A, Heward C (2004) Vitamin E and immunity after the Kona triathlon world championship. Med Sci Sports Exerc 36: 1328-1335

Noakes TD (1987) Effect of exercise on serum enzyme activities in humans. Sports Med 4:245-267

Overgaard K, Fredsted A, Hyldal A, Ingemann-Hansen T, Gissel H, Clausen T (2004) Effects of running distance and training on Ca2+ content and damage in human muscle. Med Sci Sports Exerc 36:821-829

Place N, Lepers R, Deley G, Millet GY (2004) Time course of neuromuscular alterations during a prolonged running exercise. Med Sci Sports Exerc 36(8):1347-1356

Santalla A, Naranjo J, Terrados N (2009) Muscle efficiency improves over time in world-class cyclists. Med Sci Sports Exerc 41:1096-1101

Sharwood KA, Collins M, Goedecke JH, Wilson G, Noakes TD (2004) Weight changes, medical complications, and performance during an Ironman triathlon. Br J Sports Med 38:718-724

Shima N, McNeil CJ, Rice CL (2007) Mechanomyographic and electromyographic responses to stimulated and voluntary contractions in the dorsiflexors of young and old men. Muscle Nerve 35:371-378

Stearns RL, Casa DJ, Lopez RM, McDermott BP, Gunio MS, Decher NR, Seruggs IC, West AE, Armstrong LE, Maresh CM (2009) Influence of hydration status on pacing during trail running in the heat. J Strength Cond Res 23:2533-2541

Sultana F, Brisswalter J, Lepers R, Hausswirth C, Bernard T (2008) Effects of age and gender on Olympic triathlon performances. Sci Sports 23:130-135

Suzuki K, Peake J, Nosaka K, Okutsu M, Abbiss CR, Surriano R, Bishop D, Quod MJ, Lee H, Martin DT, Laursen P13 (2006) Changes in markers of muscle damage, inflammation and HSP70 after an Ironman triathlon race. Eur J Appl Physiol 98:525-534

Tanaka H, Seals DR (2008) Endurance exercise performance in masters athletes: age-associated changes and underlying physiological mechanisms. J Physiol 586(1):56-63 
Tarpenning KM, Hamilton-Wessler M, Wiswell RA, Hawkins SA (2004) Endurance training delays age of decline in leg strength and muscle morphology. Med Sci Sports Exerc 36:74-78

Thompson LV (2009) Age-related muscle dysfunction. Exp Gerontol 44:106-111

Warren GL, Lowe DA, Armstrong RB (1999) Measurement tools used in the study of eccentric contraction-induced injury. Sports Med 27(1):43-59

Wasserman K, Whipp BJ, Koyl SN, Beaver WL (1973) Anaerobic threshold and respiratory gas exchange during exercise. J Appl Physiol 35:236-243

Westerblad H, Lee JA, Lannergren J, Allen DG (1991) Cellular mechanisms of fatigue in skeletal muscle. Am J Physiol 261:C195-C209

\section{FIGURES}

Fig. 1 A schematic representation of the experimental protocol: $\mathrm{V}_{2 \max }$ and $V T$ are the incremental cycling exercise aimed at determining maximal oxygen uptake and ventilatory threshold. CTRL denominates the control cycling exercise, while $M V C$ represents maximal voluntary contraction and neuromuscular tests

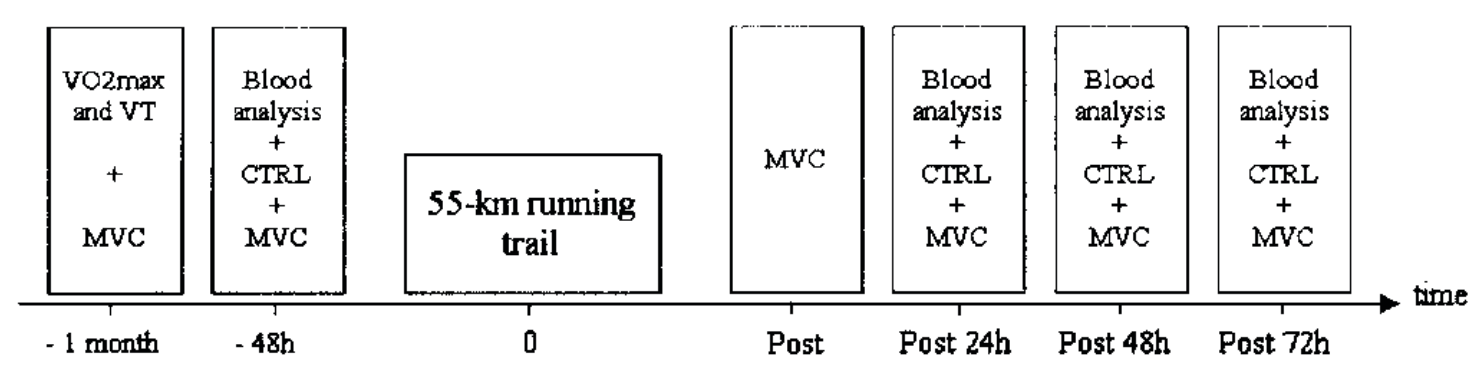


Fig. 2 Changes in knee extensors' maximal isometric strength for young and master athletes before (Pre) and $1 \mathrm{~h}$ (Post), $24 \mathrm{~h}$ (Post 24), $48 \mathrm{~h}$ (Post 48) and $72 \mathrm{~h}$ (Post 72) after the intervention. *Significantly different from pre-exercise $(P<0.05) ;{ }^{\dagger}$ significantly different from young $(P<0.05)$

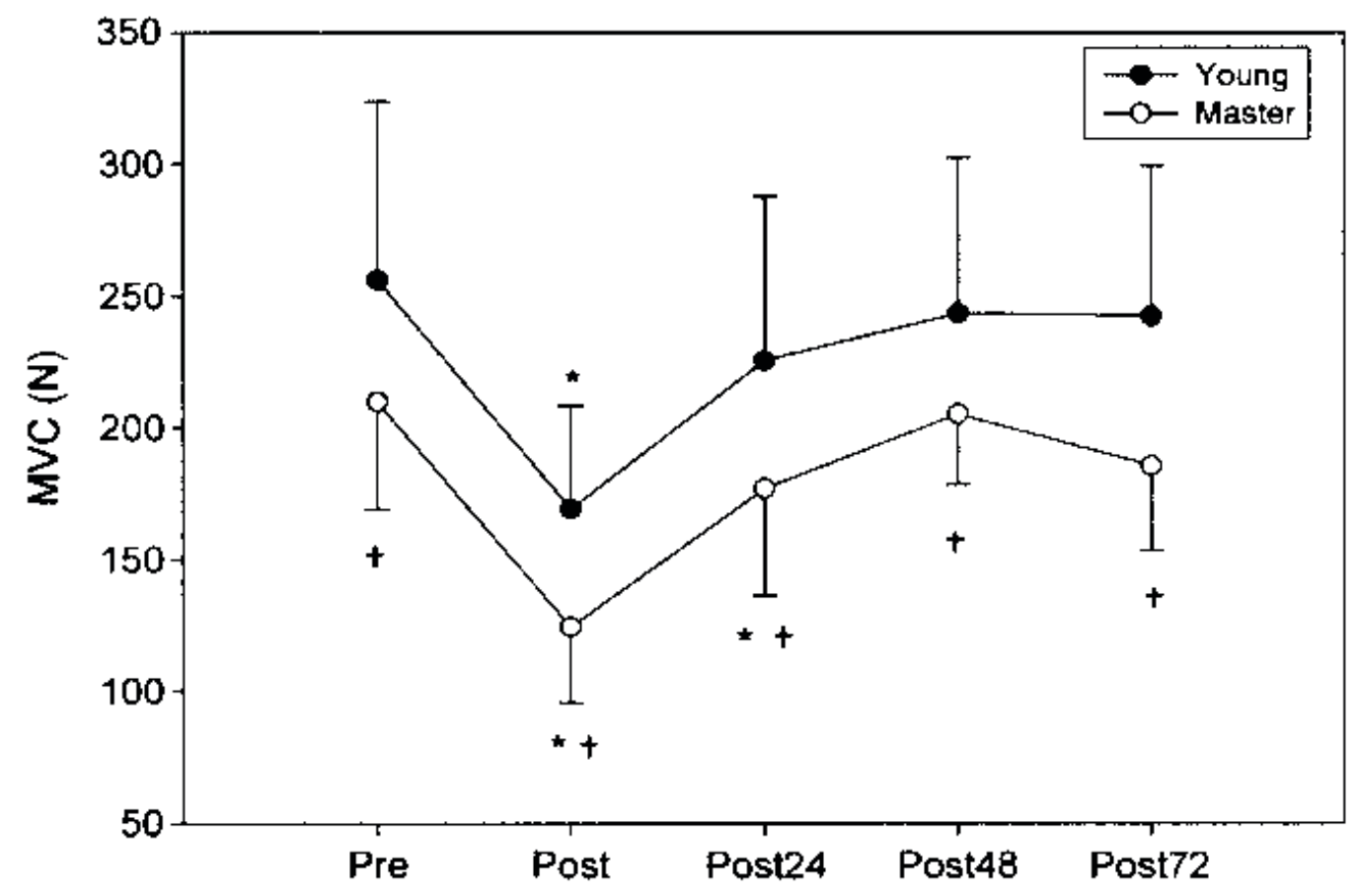

Table 1 General characteristics and performance parameters of subjects

\begin{tabular}{|l|c|c|}
\hline & Young $(\mathrm{n}=10)$ & Master $(\mathrm{n}=13)$ \\
\hline Age $($ years $)$ & $30.5(7.0)$ & $45.9(5.9)$ \\
\hline Height $(\mathrm{m})$ & $1.8(0.0)$ & $1.8(0.1)$ \\
\hline Weight $(\mathrm{kg})$ & $70.6(5.5)$ & $70.7(8.1)$ \\
\hline $\mathrm{VO}_{2}$ peak $(\mathrm{ml} / \mathrm{min} / \mathrm{kg})$ & $58.8(6.5)$ & $55.0(5.8)$ \\
\hline Marathon record (hours:min) & $03: 02(00: 26)$ & $02: 58(00: 13)$ \\
\hline $\begin{array}{l}\text { Time to complete intervention } \\
\text { (hours:min) }\end{array}$ & $06: 42(00: 51)$ & $06: 51(00: 47)$ \\
\hline
\end{tabular}

Mean (SD) values are shown 
Table 2 Twitch and M-wave parameters of the vastus lateralis muscle before (Pre), and $1 \mathrm{~h}$ (Post), $24 \mathrm{~h}$ (Post 24), $48 \mathrm{~h}$ (Post 48) and $72 \mathrm{~h}($ (Po; 72) alter the race

\begin{tabular}{|l|l|l|l|l|l|l|}
\hline Variable & & Pre & Post & Post 24 & Post 48 & Post 72 \\
\hline Twitch & & & & & & \\
\hline Pt $(\mathrm{N})$ & Young & $36(9)$ & $35(11)$ & $29(11)^{*}$ & $34(9)^{\dagger}$ & $35(12)^{\dagger}$ \\
\hline & Master & $36(11)$ & $34(12)$ & $27(12)^{*}$ & $28(08)^{*}$ & $29(12)^{*}$ \\
\hline $\mathrm{Ct}(\mathrm{ms})$ & Young & $63.3(13.7)$ & $63.4(10.6)$ & $68.8(11.2)^{*}$ & $64.7(9.5)^{\dagger}$ & $66.9(10.3)$ \\
\hline & Master & $61.3(15.6)$ & $64.9(17.4)$ & $71.1(12.9)^{*}$ & $73.2(10.8)^{*}$ & $76.2(12.7)$ \\
\hline M-Wave & & & & & & \\
\hline PPA $(\mathrm{mV})$ & Young & $3.5(1.4)$ & $3.6(1.6)$ & $3.9(1.5)$ & $3.4(1.7)$ & $3.0(1.4)$ \\
\hline & Master & $3.4(1.5)$ & $3.1(1.3)$ & $3.1(1.5)$ & $2.4(1.4)^{*}$ & $2.3(0.7)^{*}$ \\
\hline PPD $(\mathrm{ms})$ & Young & $7.6(1.5)$ & $9.2(1.2)^{*}$ & $7.0(2.2)^{\dagger}$ & $7.0(2.5)$ & $7.3(2.8)$ \\
\hline & Master & $7.9(1.5)$ & $9.5(2.5)^{*}$ & $9.3(2.8)^{*}$ & $7.8(2.7)$ & $7.6(3.3)$ \\
\hline
\end{tabular}

Mean (SD) values of 10 young and 13 master athletes are shown

$\boldsymbol{P t}$ peak twitch, $C t$ contraction time, $\boldsymbol{H} \boldsymbol{R} \boldsymbol{t}$ half-relaxation time, $\boldsymbol{P P \boldsymbol { A }}$ peak-to-peak amplitude, $\boldsymbol{P P D}$ peakto-peak duration

* Significantly different from pre-exercise $(\boldsymbol{P}<0.05)$

${ }^{\dagger}$ Significantly different from master $(\boldsymbol{P}<0.05)$

Table 3 Changes in muscle damage indicating blond markers for young and master athletes before (Pre), $24 \mathrm{~h}$ (Post $24 \mathrm{~h}$ ), $48 \mathrm{~h}$ (Post $48 \mathrm{~h}$ ) and $72 \mathrm{~h}$ (Post $72 \mathrm{~h}$ ) after the race

\begin{tabular}{|l|l|l|l|l|l|l|}
\hline Variable & Group & $\begin{array}{l}\text { Normal } \\
\text { range }\end{array}$ & Pre & Post 24h & Post 48 h & Post 72 h \\
\hline CK(U/1) & Young & $50-230$ & $135(26)$ & $1,470(565)^{* \dagger}$ & $909(303)^{*}$ & $430(251)^{*}$ \\
\hline & Master & $50-230$ & $138(107)$ & $1,559(593)^{*}$ & $920(298)^{*}$ & $531(271)^{*}$ \\
\hline LDH(U/1) & Young & $120-245$ & $229(52)$ & $528(164)^{*}$ & $453(65)^{*}$ & $410(65)^{*}$ \\
\hline & Master & $120-245$ & $194(63)$ & $482(142)^{*}$ & $468(105)^{*}$ & $473(165)^{*}$ \\
\hline
\end{tabular}

$\boldsymbol{C K}$ creatine kinase, $\boldsymbol{L D H}$ lactate dehydrogenase

* Significantly different from pre-exercise $(P<0.05)$

${ }^{\dagger}$ Significantly different from masters $(P<0.05)$ 
Table 4 Changes in efficiency, ventilation and cycling cadence for young and masters during cycling exercises performed before (Pre), $24 \mathrm{~h}$ (Post 24), $48 \mathrm{~h}$ (Post 48) and $52 \mathrm{~h}$ (Post 52) after the race

\begin{tabular}{|l|l|l|l|l|l|}
\hline \multicolumn{1}{|c|}{ Variable } & & \multicolumn{1}{c|}{ Pre } & \multicolumn{1}{c|}{ Post 24 } & \multicolumn{1}{c|}{ Post 48 } & \multicolumn{1}{c|}{ Post 72 } \\
\hline GE (bpm) & Young & $19.6(2.4)$ & $18.8(1.5)^{*}$ & $18.2(2.9)^{*}$ & $19(2.1)^{*}$ \\
\hline & Master & $19.9(1.5)$ & $19.1(1.5)^{*}$ & $18(1.9)^{*}$ & $18.8(1.5)^{*}$ \\
\hline DE(l/min) & Young & $25.1(5)$ & $22.5(2.6)^{\dagger}$ & $25.4(5.6)^{\dagger}$ & $25.4(5.8)^{\dagger}$ \\
\hline & Master & $22.5(2.6)$ & $20.1(2.4)^{*}$ & $19.9(5.7)^{*}$ & $20.6(5.2)^{*}$ \\
\hline VE( $(/ m i n)$ & Young & $54(9)^{\dagger}$ & $81(12)^{* \dagger}$ & $85(15)^{*}$ & $82(15)^{*^{\dagger}}$ \\
\hline & Master & $85(11)$ & $90(11)^{*}$ & $94(14)^{*}$ & $90(14)^{*}$ \\
\hline Cad $(\mathrm{rpm})$ & Young & $85(8)$ & $86(12)^{* \dagger}$ & $92(15)^{*^{\dagger}}$ & $95(15)^{*^{\dagger}}$ \\
\hline & Master & $82(9)$ & $80(8)$ & $85(10)^{*}$ & $90(8)^{*}$ \\
\hline
\end{tabular}

Mean (SD) values of 10 young and 15 master athletes are shown

$G E$ gross efficiency, $D E$ delta efficiency, $V E$ minute ventilation, $C a d$ pedaling cadence

* Significantly different from pre-exercise $(P<0.05)$

$\dagger$ Significantly different from masters $(P<0.05)$ 